\title{
Romance and sex before marriage among young women and men in Andhra Pradesh
}

International Institute for Population Sciences (IIPS)

Population Council

Follow this and additional works at: https://knowledgecommons.popcouncil.org/departments_sbsr-pgy

Part of the Demography, Population, and Ecology Commons, Family, Life Course, and Society Commons, Gender and Sexuality Commons, International Public Health Commons, Public Health Education and Promotion Commons, and the Women's Health Commons How does access to this work benefit you? Let us know!

\section{Recommended Citation}

International Institute for Population Sciences (IIPS) and Population Council. 2010. "Romance and sex before marriage among young women and men in Andhra Pradesh," Youth in India: Situation and Needs Policy Brief no. 29. Mumbai: IIPS. 


\section{Romance and sex before marriage among young women and men in Andhra Pradesh}

\begin{abstract}
Pre-marital partnerships among youth, including those that do not involve sexual relations, are widely discouraged in India. Indeed, there is a widespread perception that youth do not find opportunities to mix and form romantic relationships and that pre-marital sex is negligible. These kinds of perceptions are often cited to argue against the provision of sex or family life education and sexual and reproductive health services to unmarried young people.
\end{abstract}

While several small and unrepresentative studies have noted that sizeable proportions of young people, particularly young men, are sexually active before marriage, ${ }^{\text {abcdef }}$ the lack of rigorous and representative evidence on the levels and patterns of intimate partnerships among youth continues to hamper designing of appropriate policies and programmes to address youth sexual health needs. This policy brief documents the extent of pre-marital romantic and sexual relations experienced by young men and women in Andhra Pradesh, and the extent to which pre-marital sexual experiences among young people are informed, safe and wanted.

\section{The study}

Data are drawn from the Youth in India: Situation and Needs study, a sub-nationally representative study undertaken for the first time in India of key transitions experienced by young people in six states of India. The study included a representative survey of young people in both rural and urban settings. Respondents included unmarried women and men and married women aged 15-24 and, in view of the paucity of married men in these ages, married men aged 15-29.
In Andhra Pradesh, the survey was conducted in 2007-08. A total of 8,330 married and unmarried young men and women were interviewed in the survey. These included 1,405 married young men, 2,077 unmarried young men, 2,330 married young women and 2,518 unmarried young women. This brief is based on data obtained from 2,479 young men and 4,848 women aged 15-24.

Acknowledging that young people may have been reluctant to disclose pre-marital sexual experiences, the Youth Study included a number of approaches to elicit data on sexual behaviour. Questions were posed both in a face-to-face interview format as well as an anonymous format in which youth were asked to mark a blank card indicating whether or not they had ever experienced pre-marital sex, place the card in an envelope, seal it and return it to the interviewer. Envelopes were opened only at study headquarters. In addition, youth were also asked to report, anonymously, the pre-marital sexual experiences of their peers.

Opportunities to form pre-marital romantic relationships do exist for young people Despite norms prohibiting pre-marital opposite-sex mixing, findings indicate that opportunities to form pre-marital romantic relationships did exist for young people. Findings suggest that $23 \%$ of young men and women had either been approached by or had approached a person of the opposite sex to form a romantic liaison. Moreover, $17 \%$ of young men and $11 \%$ of young women acknowledged the experience of a romantic partnership before marriage. Where romantic relationships occurred, they were

a Abraham, L. 2001. "Redrawing the lakshman rekha: Gender differences and cultural constructions in youth sexuality in urban India," South Asia 24:133-56.

b Abraham, L. 2002. "Bhai-behen, true love, time pass: Friendships and sexual partnerships among youth in an Indian metropolis," Culture, Health, and Sexuality 4(3):337-53.

c Abraham, L. and K.A. Kumar. 1999. "Sexual experiences and their correlates among college students in Mumbai city, India," International Family Planning Perspectives 25(3):139-46.

d Alexander, M., L. Garda, S. Kanade et al. 2006a. Formation of Partnerships among Young Women and Men in Pune District, Maharashtra. New Delhi: Population Council.

${ }^{\text {e }}$ Alexander, M., L. Garda, S. Kanade et al. 2006b. "Romance and sex: Pre-marital partnership formation among young women and men, Pune district, India," Reproductive Health Matters 14(28):144-55.

${ }^{\mathrm{f}}$ Awasthi, S., M. Nichter and V.K. Pande. 2000. "Developing an interactive STD prevention programme for youth: Lessons from a north Indian slum," Studies in Family Planning 31(2):138-50. 
Extent of pre-marital romantic relationships: Percentage of youth who reported a romantic partner

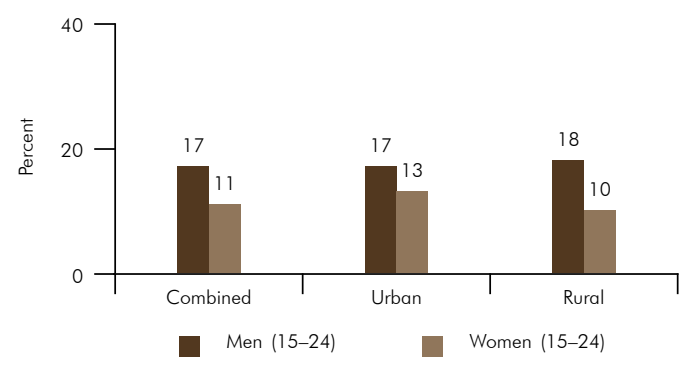

usually hidden from parents but not from peers. Among those who had experienced a pre-marital romantic partnership, almost all young men $(94 \%)$ and four in five young women (82\%) reported that their peers were aware of their relationship. Relatively fewer youth reported that their parents were aware of their relationship: $40 \%$ of young men and $59 \%$ of young women reported that their parents were aware of their relationship.

The majority of young men and women who reported a pre-marital romantic partnership had engaged in a range of intimate behaviours with the romantic partner, from holding hands to kissing on the lips to sexual intercourse. For example, $95 \%$ of young men and $82 \%$ of young women had held hands with their romantic partner. Consistently fewer reported progressively more intimate behaviours: about four-fifths of young men (77-82\%) and almost two-thirds of young women $(63 \%)$ had hugged or kissed their romantic partner, and close to half of young men and women $(46 \%)$ had engaged in sex with their romantic partner.

Findings also show that relatively large proportions of young men and women engaged in a romantic relationship with expectations of marriage with the romantic partner. Even so, young women were considerably more likely than young men to have expected a romantic relationship to lead to marriage. Of those who reported a pre-marital romantic relationship, almost all young women (92\%) compared to fewer than three-quarters of young men $(72 \%)$ intended to marry their romantic partner.

\section{Engaging in sex before marriage is not} unknown

The Youth Study inquired about pre-marital sex both with romantic partners and in other situations, for example, casual, paid and forced. Findings indicate that one in six young men $(16 \%)$ and one in twenty young women $(6 \%)$ had engaged in pre-marital sex within romantic and/or other partnerships. Rural young men were somewhat more likely than their urban counterparts to have experienced pre-marital sex ( $17 \%$ of rural young men compared to $14 \%$ of urban young men). In contrast, rural-urban differences were not observed among young women (6\% each). Notably, differences by education were negligible, suggesting that better educated youth were about as likely as their less educated counterparts to have engaged in pre-marital sex.

Extent of pre-marital sexual experiences: Percentage of youth who reported having engaged in pre-marital sex

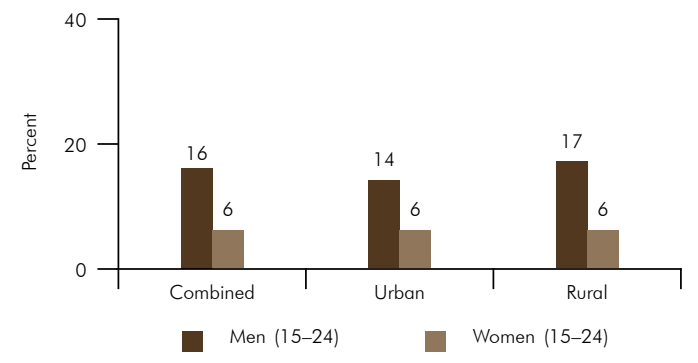

Progression of physical intimacy among youth who reported pre-marital romantic partnerships

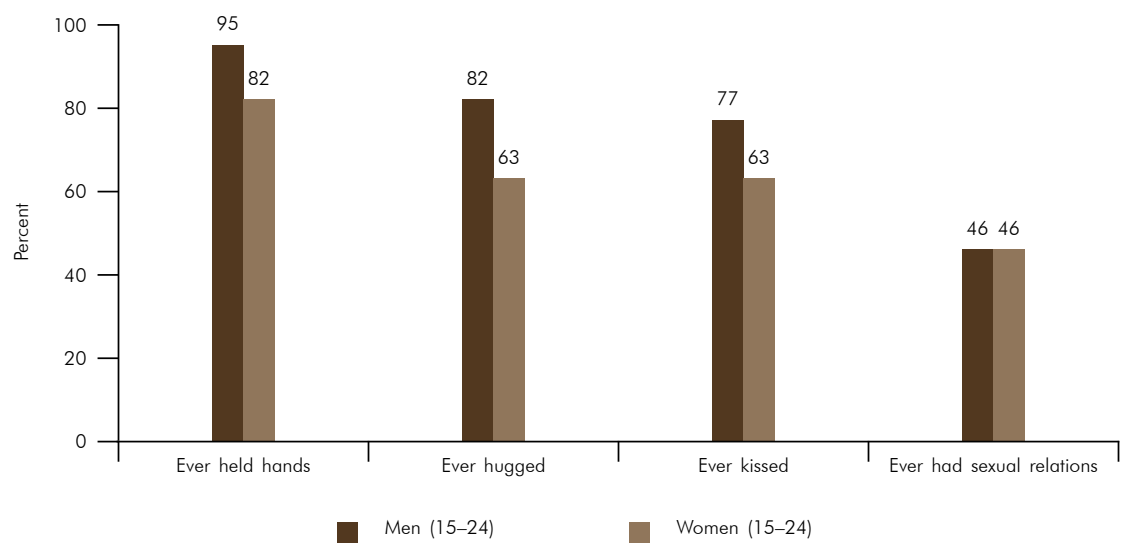


Many young people initiate pre-marital sex uninformed

Findings also confirm that many young people had initiated sexual activity uninformed. For example, just half of young men $(52 \%)$ and three-quarters of young women $(72 \%)$ who were sexually experienced before marriage knew that a woman can get pregnant at first sex.

In-depth awareness of contraceptive methods was not universal among the sexually experienced, but was particularly limited among sexually experienced young women. For example, 98\% of young men who were sexually experienced before marriage had heard of condoms and $92 \%$ knew that one condom can be used for only one sexual act. In contrast, just $80 \%$ of young women had heard about condoms and far fewer-38\%-knew that one condom can be used for only one sexual act.

In view of the state's focus on spreading information about HIV/AIDs, it is no surprise that almost all sexually experienced youth had heard of HIV (98-99\%). However, comprehensive knowledge of HIV/AIDS ${ }^{1}$ was limited. Indeed, only $64 \%$ of sexually experienced young men and 39\% of sexually experienced young women had comprehensive knowledge of HIV/AIDS. Worse, fewer than half $(46 \%)$ of sexually experienced young men and just one-fifth (19\%) of sexually experienced young women had heard of STIs other than HIV.

Corresponding with their lack of awareness of sexual and reproductive health matters, relatively few youth reported exposure to sexuality or family life education. Indeed, just $28-29 \%$ of sexually experienced young men and women had ever received sexuality or family life education.
Engaging in pre-marital sex with multiple partners is not uncommon

Findings show that where pre-marital sex was experienced, it was by and large unsafe. Of young men who were sexually experienced before marriage, as many as $22 \%$ had sex with two or more partners. In contrast, fewer sexually experienced young women-7\%-reported that they had engaged in pre-marital sexual relations with multiple partners.

\section{Consistent condom use within pre-marital sexual relationships is rare}

Few sexually experienced youth reported consistent condom use. Indeed, just $17 \%$ of young men who had experienced pre-marital sex reported that they had always used a condom. In contrast, hardly any sexually experienced young women $(2 \%)$ so reported.

Of those who had experienced pre-marital sex, percentage of youth who reported multiple partnerships and consistent condom use

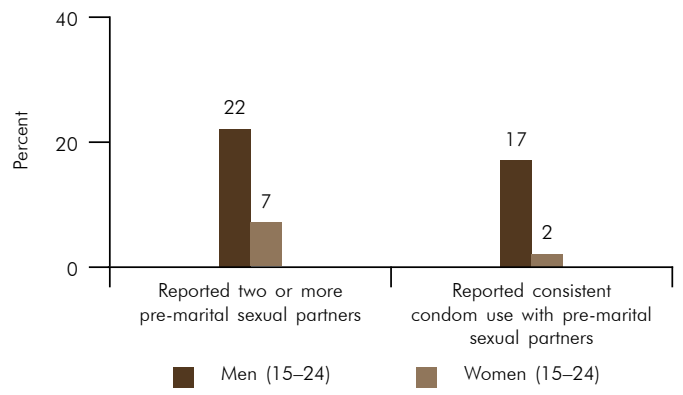

Pre-marital sex is not always consensual Pre-marital sexual experience was non-consensual for substantial minorities of young people, particularly young women. Of those who had engaged in pre-marital sexual relations, $3 \%$ of young men and $8 \%$ of young women reported that
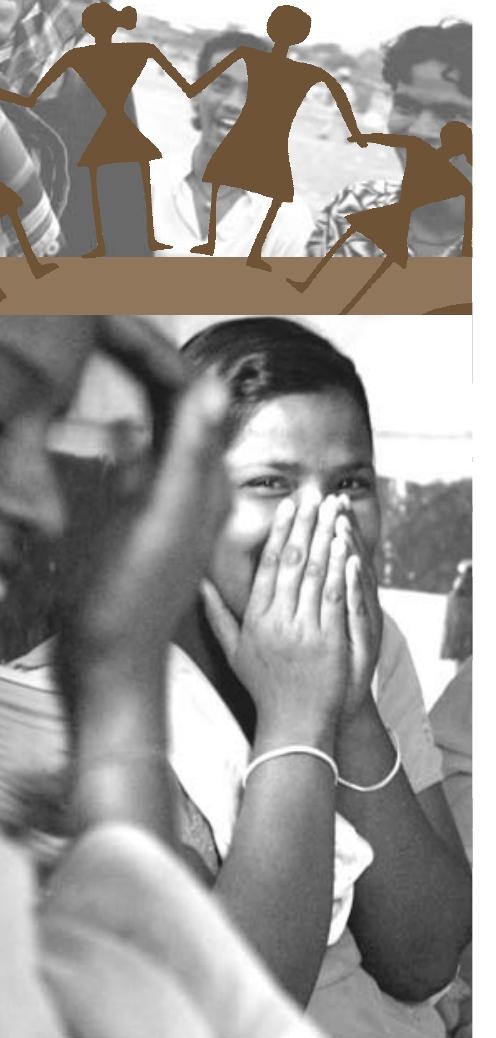

\section{Of those who had experienced pre-marital sex, percentage of youth who reported in-depth}

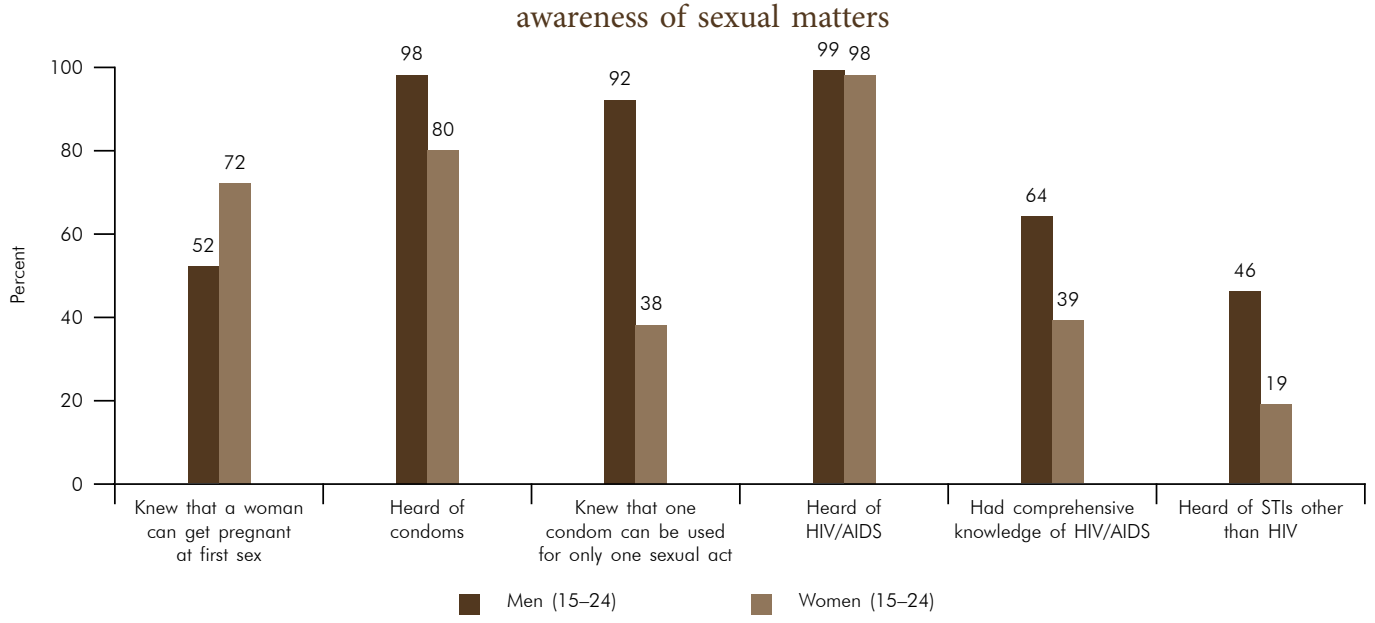

${ }^{1}$ Comprehensive awareness is defined as knowledge of two ways of preventing HIV (specifically, condom use and single partner relations), rejection of common misconceptions about HIV transmission (namely, that HIV can be transmitted through mosquito bites, sharing food or hugging) and awareness that one cannot tell by looking at a person whether he or she has HIV. 
Of those who had experienced pre-marital sex, percentage of youth who reported non-consensual sexual experiences

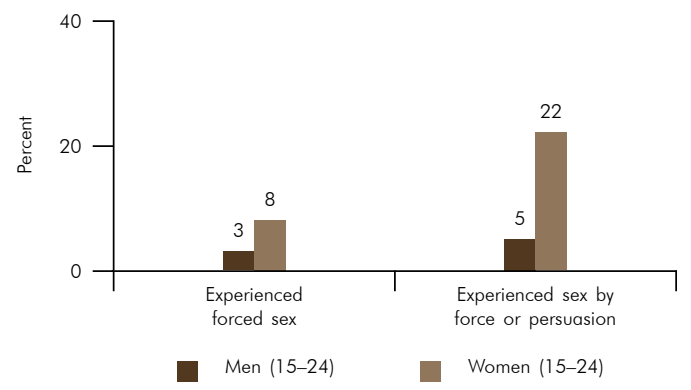

they were forced to engage in sex. Few young men (5\%) and over one-fifth of young women $(22 \%)$ reported that they were either persuaded or forced to engage in sex.

\section{Implications for programmes}

Findings presented above clearly highlight that many young men and a small minority of young women in Andhra Pradesh had engaged in sex before marriage and that many of them had initiated sexual activities uninformed, had engaged in unsafe relations and/or had experienced unwanted relations. The better educated were as likely as the less educated to have engaged in sexual relations. Policies and programmes can no longer assume that pre-marital sex does not exist in our culture, rather they must ensure that sexual relations, should they occur, are informed, safe and wanted. Several steps are urgently needed.

\section{Provide family life or sex education for those in school and out of school}

Youth must be equipped with information on physical maturation, relationship matters, risk and protective sexual behaviours, including the role of condoms. They must also be made aware of where they can obtain information, counselling and services. Sex and family life education programmes are needed that build awareness in ways that meet the needs of all youth, both those in school and out-of-school. Programmes are needed that respond to, rather than obfuscate, questions youth raise on sexual health. Such information will allow youth to better assess whether to delay sexual relations, on the one hand and to ensure that relations, if undertaken, are safe, on the other.

Findings that relatively few youth had been exposed to family life or sex education emphasise the need for increased investment by the state in promoting such education for youth in school and out of school.

Equally important is the acquisition of life skills that enable youth to put information into practice. Findings underscore the need for programmes that focus on building young people's skills in negotiating safe sex and communicating with partners, and that encourage young people to break down gender stereotypes and relate to each other as equals.

\section{Reorient service provision to address the sexual and reproductive health needs of unmarried youth}

At the same time, programmes must make available appropriate family planning and infection prevention services for unmarried young men and women in a manner acceptable to them. The National Adolescent Reproductive and Sexual Health strategy of the Reproductive and Child Health $(\mathrm{RCH})$ programme has recognised the need to address the needs of the unmarried, but much remains to be done in Andhra Pradesh in terms of reorienting providers to include the unmarried in the ambit of services provided and to shed their own misgivings about addressing the needs of sexually active unmarried youth. 